Universidad Nacional Experimental Francisco de Miranda (UNEFM). Santa Ana de Coro. Venezuela

María Fernanda Jimbo-Amaya; Edwin Joselito Vásquez-Erazo;

Maira Elizabeth Ramón-Naranjo

DOI $10.35381 / \mathrm{cm} . v 7 i 12.429$

\title{
Spin Off en el entorno Universitario: Caracterización y Determinantes para su
} creación

Spin Off in the University environment: Characterization and Determinants for its creation

\author{
María Fernanda Jimbo-Amaya \\ mijmbo@ucacue.edu.ec \\ Universidad Católica de Cuenca, Cuenca \\ Ecuador \\ https://orcid.org/0000-0002-0401-1722 \\ Edwin Joselito Vásquez-Erazo \\ evasqueze@ucacue.edu.ec \\ Universidad Católica de Cuenca, Cuenca \\ Ecuador \\ https://orcid.org/0000-0001-9817-6773 \\ Maira Elizabeth Ramón-Naranjo \\ meramonn@ucacue.edu.ec \\ Universidad Católica de Cuenca, Cuenca \\ Ecuador \\ https://orcid.org/0000-0002-2691-1844
}

Recibido: 01 de octubre de 2020

Aprobado: 15 de diciembre de 2020 


\author{
CIENCIAMATRIA \\ Revista Interdisciplinaria de Humanidades, Educación, Ciencia y Tecnología \\ Año VII. Vol. VII. N¹2. Enero - Junio. 2021 \\ Hecho el depósito de ley: pp201602FA4721 \\ ISSN-L: 2542-3029; ISSN: 2610-802X \\ Universidad Nacional Experimental Francisco de Miranda (UNEFM). Santa Ana de Coro. Venezuela \\ María Fernanda Jimbo-Amaya; Edwin Joselito Vásquez-Erazo; \\ Maira Elizabeth Ramón-Naranjo
}

\title{
RESUMEN
}

El presente estudio investigativo tiene como finalidad diseñar una propuesta de un modelo de generación de Spin-Off en el entorno universitario, dado el ecosistema emprendedor con el que cuenta la Universidad Católica de Cuenca. Se realizó en base a una metodología descriptivo con diseño de campo no experimental. el $75 \%$ de los docentes manifestó que el emprendimiento debe incorporarse en las mallas académicas debido a su importancia. Es importante incentivar el emprendimiento desde la academia, esto es mediante la ejecución de proyectos presentados por estudiantes en apoyo conjunto con docentes investigadores los mismos que permitirán desarrollar competencias en su desempeño profesional, es importante recalcar que una universidad necesita desarrollar procesos innovadores en su formación para ser considerada emprendedora.

Descriptores: Universidad; sector público; sector privado; investigación y desarrollo; financiación de la educación. (Palabras tomadas del Tesauro UNESCO).

\begin{abstract}
The purpose of this research study is to design a proposal for a Spin-Off generation model in the university environment, given the entrepreneurial ecosystem that the Universidad Católica de Cuenca has. It was carried out based on a descriptive methodology with a non-experimental field design. $75 \%$ of teachers stated that entrepreneurship should be incorporated into academic networks due to its importance. It is important to encourage entrepreneurship from the academy, this is through the execution of projects presented by students in joint support with research professors who will allow them to develop skills in their professional performance, it is important to emphasize that a university needs to develop innovative processes in their training to be considered an entrepreneur.
\end{abstract}

Descriptors: Universities; Public sector; Private sector; Research and development; Educational finance. (Words taken from UNESCO Thesaurus). 


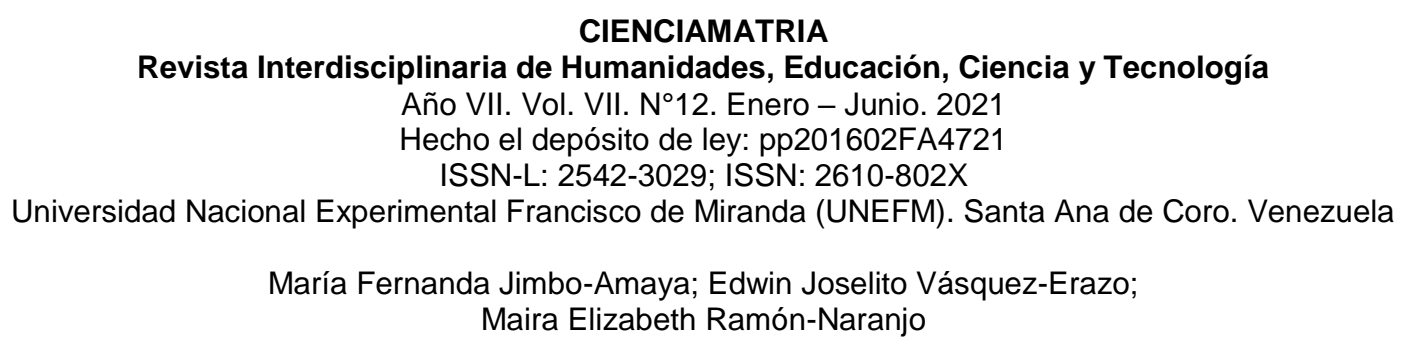

\section{INTRODUCCIÓN}

La puesta en marcha de empresas que se originan dentro del ámbito académico denominado Spin Off y que nacen de un proceso de incubación empresarial a nivel universitario, son iniciativas originadas por catedráticos y estudiantes dedicados a la investigación científica y tecnológica cuya particularidad es la búsqueda e innovación de nuevos procesos, productos/servicios a partir de los conocimientos adquiridos, tomando siempre en consideración las necesidades que presentan tanto las empresas como la sociedad en general.

Las incubadoras de empresas se han convertido en un instrumento de suma importancia por cuanto ayuda a desarrollar la economía de un país, a través de la asesoría para la implementación de una idea de negocios, el aumento del empleo y la creación de un entorno favorable.

En la década de los 50 en Silicón Valley California las incubadoras surgieron con la creación de un parque industrial y tecnológico (Stanford Research Park), el cual tenía como objetivo esencial promover transferencia tecnológica desde las Universidades hacia las empresas, para la década de los 70 se desarrolló la estructura de las incubadoras actuales a partir de la implementación de programas formales de incubación en el país de Estados Unidos, es así que desde entonces se han utilizado las incubadoras como herramienta para el desarrollo socio-económico en varios países (MopositaYanchatuña, et al., 2018).

En América Latina por el periodo de los años 80 se han aumentado las acciones que las universidades realizan con la finalidad de generar vínculos con el sector productivo, es así que en el año 90 ya se asociado mecanismos para la creación y promoción de emprendimientos productivos innovadores. Se ha podido observar que en Latinoamérica las incubadoras de empresas han tenido mayor apogeo en los centros de educación superior, es así que las primeras publicaciones investigativas con relación a incubadoras fueron de carácter descriptivos es decir sin ninguna base conceptual, centrando su 


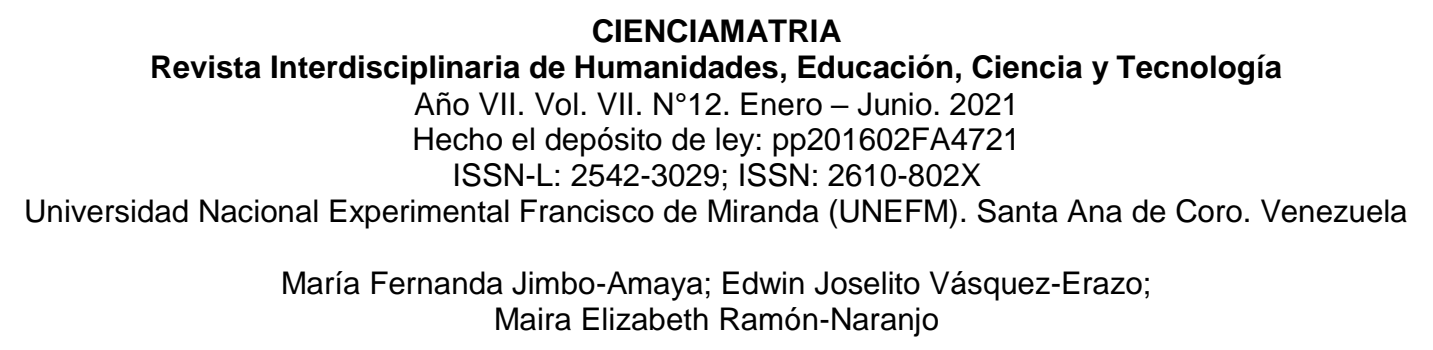

investigación en el análisis de la incubadora como unidad de negocio y no en sus características.

Las universidades ecuatorianas hoy en día enfrentan diferentes retos, el más importante radica en la calidad de la educación que brinda a sus estudiantes, es decir que permita formar profesionales emprendedores altamente competitivos que ayuden a brindar soluciones a los problemas del entorno y de esta manera contribuyan con el desarrollo económico y social.

Las Instituciones de Educación Superior (IES), no vienen desarrollando contextos adecuados para la generación de Spin-Off por cuanto ha existido escaso aporte en la generación de incubadoras, debido a que muy pocas carreras se orientan al saber hacer de la profesión y no se generan ideas emprendedoras en las aulas, lo que limita el desarrollo productivo del sector de incidencia y no hay aporte para el fortalecimiento de emprendimientos en la zona.

Por ello es preciso diseñar una propuesta de un modelo de generación de Spin-Off en el entorno universitario, dado el ecosistema emprendedor con el que cuenta la Universidad Católica de Cuenca.

\section{Referencial Teórico}

\section{La Gestión De Emprendimiento en las Universidades}

Los procesos de formación e investigación son gestiones que vienen cumpliendo las Instituciones de Educación Superior (IES) a más de promover otras nuevas que permitan reflejar la pertinencia, mediante una gestión social del conocimiento visualizado mediante contribuciones económicas, sociales y culturales de su evolución (Trippl, et al., 2015).

En este sentido la pertinencia de las instituciones de educación superior (IES) debe dar respuesta a las demandas sociales, priorizando la construcción de lo público y la identidad ciudadana con la finalidad de aportar al crecimiento empresarial y al desarrollo productivo e innovador con nuevas ideas de negocios. De tal manera que se pueda decir que estas nuevas gestiones obligan a las universidades ecuatorianas a replantear su 


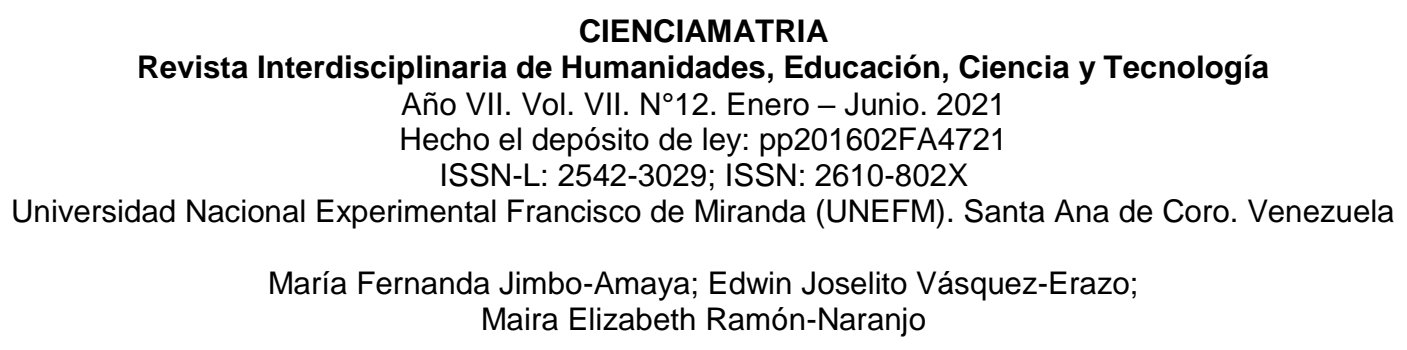

accionar y responder eficiente y eficazmente este nuevo reto que tiene que enfrentar la educación superior.

Por otro lado (Romero-Paz, et al., 2020), las Instituciones de Educación Superior (IES) están encargadas de producir conocimiento científico-tecnológico, actualmente son revalorizadas en función de su potencial contribución al desarrollo económico de la sociedad, se puede decir entonces que las funciones ejercidas por las (IES) han ido evolucionando con el transcurso del tiempo, esto es adaptándose a la evolución y necesidades que presenta la sociedad y al desarrollo de la economía en general. Según (Freire, 2015), es necesario brindar una atención adecuada y exhaustiva a las enseñanzas universitarias, incluyendo tareas de información y asesoramiento para que los estudiantes se inicien en el campo del emprendimiento.

Cuando se habla de cultura de emprendimiento, es necesario pensar en la función específica de las Instituciones de Educación Superior, cuyo elemento principal es formar profesionales en las diferentes áreas o especialidades, sin embargo es preciso que docentes y estudiantes desarrollen procesos pragmáticos que desencadene en la generación de emprendimientos y con ello la creación de spin-off dentro de la instituciones educativas, naturalmente involucrando a la sociedad para determinar sus necesidades y problemas, aportando así a la estructura de ecosistemas de emprendimiento donde participen los diferentes actores sociales, gobierno, universidad, entidades financieras y los emprendedores, construyendo un sistema de apoyo, investigación e innovación que generen resultados importantes en la sociedad.

Hoy en día se está trabajando en el fortalecimiento del vínculo universidad-empresa, asumiendo un rol cada vez más protagónico, dado que han pasado de ser universidades centradas en la formación académica a ser universidades emprendedoras, esto es semilleros en la creación de nuevas empresas denominadas Spin-Off, de la misma forma es más frecuente localizar iniciativas académicas que estimulan la creación de nuevas empresas cuya principal finalidad es la satisfacción de necesidades antes la escases de recursos. 


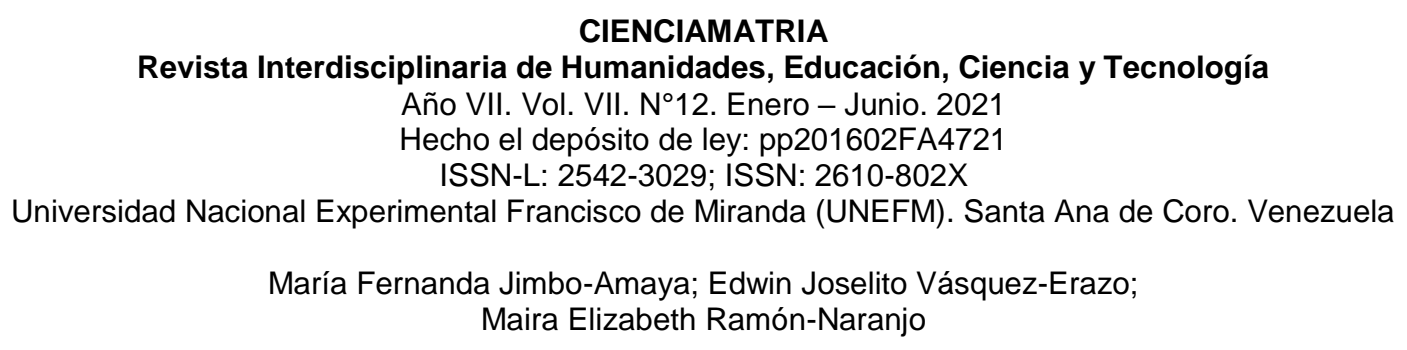

Por tanto, esta situación ha obligado a las Instituciones de Educación Superior (IES) a implementar estrategias que permitirán utilizar sus recursos en la creación de entidades que desarrollen la función de investigación, en muchos casos orientados a la generación de emprendimientos que a su vez les permita obtener beneficios económicos.

No obstante, una serie de aspectos relativos a este fenómeno son todavía poco comprendidos. Por tal razón el concepto de spin off en la práctica parece presentar cierta ambigüedad al no existir unanimidad en la terminología utilizada. Por ello, es significativo precisar el verdadero alcance del concepto de spin-off (Maridueña-Arroyave, et al., 2016). Se puede mencionar entonces, que la nueva función de las IES debe orientar su mirada a realizar procesos de investigación, desarrollo que puedan potenciar descubrimientos que generen Spin-Off de tal manera que se fortalezca el sistema productivo, satisfaciendo así las necesidades sociales. Para que una universidad sea considerada emprendedora debe cumplir ciertas acciones que se detallan a continuación según (Arata-Andreani \& Soto-Salazar, 2010), debe:

1. Contar con un equipo de investigación organizado.

2. Establecer el activo principal de la Universidad, esto es sus dominios o fortalezas en áreas específicas de investigación y desarrollo, tomando en consideración las áreas que tengan mayor posibilidad de explotarse para obtener resultados positivos.

3. Salvaguardar la propiedad intelectual y los servicios necesarios a fin de que permitan valorizar los productos en proceso de Investigación, desarrollo y comercialización.

4. Promover el desarrollo y crecimiento de spin-off a nivel de academia.

5. Estrechar la relación Universidad - Empresa - Estado.

En cuanto a la universidad tradicional su enfoque está dirigido a la formación, investigación, generación de conocimiento como ejes fundamentales de su labor institucional. Por tal razón las condiciones actuales y las características económicas y sociales de la sociedad han puesto en discusión la relación entre universidad, empresa y 


\section{CIENCIAMATRIA \\ Revista Interdisciplinaria de Humanidades, Educación, Ciencia y Tecnología \\ Año VII. Vol. VII. Nº12. Enero - Junio. 2021 \\ Hecho el depósito de ley: pp201602FA4721 \\ ISSN-L: 2542-3029; ISSN: 2610-802X \\ Universidad Nacional Experimental Francisco de Miranda (UNEFM). Santa Ana de Coro. Venezuela \\ María Fernanda Jimbo-Amaya; Edwin Joselito Vásquez-Erazo; \\ Maira Elizabeth Ramón-Naranjo}

gobierno, es por ello que debería existir un nexo entre las 3 entidades cuya finalidad sea la cooperación interinstitucional en pro del desarrollo de la sociedad y de sus economías (Campos \& Sánchez, 2006). Al respecto conviene decir que la vinculación de la universidad con el sector empresarial se debería ampliar, de modo que el apoyo sea mutuo y por ende generar nuevas estrategias de vinculación, con un enfoque de Megaplaneación (Kaufman, 2004).

En otras palabras, la cooperación entre universidades, sector empresarial y gobierno es fundamental puesto que con su aporte los universitarios pueden desarrollar sus ideas de negocios, mediante la implementación de programas e iniciativas que contribuyan con el aumento de calidad e impacto social de los conocimientos producidos en los centros de investigación (Torres-Vázquez, et al., 2020).

Una de las teorías que más impacto ha tenido es el modelo triple hélice de (Etzkowitz \& Leydesdorff, 2000), el cual implica una profunda colaboración entre Estado, Universidad e Industria a fin de que todos estos factores se conviertan en colaboradores activos y contribuyan con el desarrollo económico y social, por esta razón la interacción entre estos factores es de vital importancia siendo el camino a seguir para obtener beneficios para la sociedad en general. De acuerdo con (Beltrán-Ibarra \& Lagarda-Leyva, 2015) este modelo abarca las tres hélices que se complementan entre sí, permitiendo una relación de ganar-ganar para acelerar el crecimiento de la región (Martínez-Ortíz, et al., 2016), Manifiestan que hoy en día los ecosistemas emprendedores utilizan este modelo que permite integrar Gobierno, Academia y Sector Empresarial, pero es necesario recalcar la opinión de (Salamea \& Peña, 2015), el cual propone un cuarto elemento fundamental que define el éxito o fracaso de los emprendimientos, y son los consumidores los cuales garantizan la aceptación o rechazo de un emprendedor frente a la sociedad.

Cada región, ciudad y territorio de acuerdo a sus potencialidades e individualidades precisa adaptación de los ecosistemas en los cuales se tiene que construir ambientes favorables y confiables para el emprendedor, es así que los ecosistemas locales facilitan el surgimiento de una nueva empresa mediante el esfuerzo en su accionar. En la figura 


\section{CIENCIAMATRIA \\ Revista Interdisciplinaria de Humanidades, Educación, Ciencia y Tecnología \\ Año VII. Vol. VII. N¹2. Enero - Junio. 2021 \\ Hecho el depósito de ley: pp201602FA4721 \\ ISSN-L: 2542-3029; ISSN: 2610-802X \\ Universidad Nacional Experimental Francisco de Miranda (UNEFM). Santa Ana de Coro. Venezuela \\ María Fernanda Jimbo-Amaya; Edwin Joselito Vásquez-Erazo; \\ Maira Elizabeth Ramón-Naranjo}

1 se puede observar que el éxito de un emprendimiento surge de acciones fluidas entre de los actores del ecosistema.

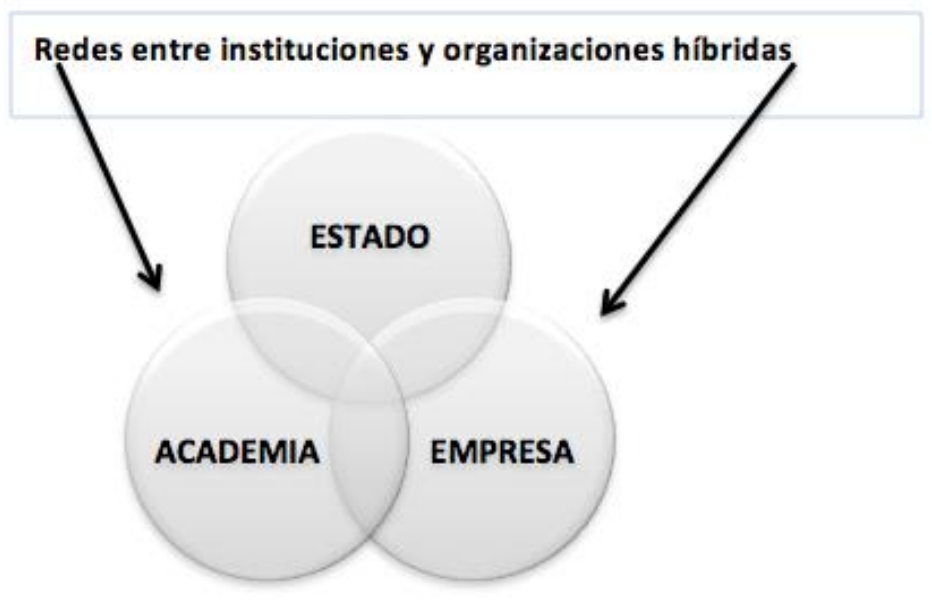

Figura 1: Modelo Triple Hélice.

Fuente: Etzkowitz \& Leydesdorff (2000).

Díez-Sáez (2014) Enfatiza la importancia que existe entre el vínculo universidad, industria y estado ya que permite la generación de mayor valor en su punto central de coincidencia, dentro de estas redes se constituyen las Spin-Off, basada en el conocimiento e investigación previamente financiada por el Estado.

\section{El Desarrollo de las Incubadoras}

Las incubadoras de empresas están enfocadas a ser organizaciones que apoyen al desarrollo de emprendimiento y creación de negocio, mitigando riesgos y aumentando las posibilidades de éxito de quienes busquen apoyo en este tipo de organización. Desde sus inicios en los años 50 cuando no eran considerados como tal las incubadoras se convirtieron en una revolución en el mundo empresarial con el objetivo de poder transferir la tecnología desarrollada hacia las empresas y crear nuevas empresas; el éxito que se 


\section{CIENCIAMATRIA \\ Revista Interdisciplinaria de Humanidades, Educación, Ciencia y Tecnología \\ Año VII. Vol. VII. N¹2. Enero - Junio. 2021 \\ Hecho el depósito de ley: pp201602FA4721 \\ ISSN-L: 2542-3029; ISSN: 2610-802X \\ Universidad Nacional Experimental Francisco de Miranda (UNEFM). Santa Ana de Coro. Venezuela \\ María Fernanda Jimbo-Amaya; Edwin Joselito Vásquez-Erazo; \\ Maira Elizabeth Ramón-Naranjo}

ha obtenido en algunos casos ha hecho que esta iniciativa fuera replicada en otras partes del mundo (León-Pérez \& Benítez-Pincay, 2019).

Por otro lado, (Perez-Palacios, 2009), indica que según expertos el $80 \%$ de las empresas que nacen en una incubadora de empresas logra el éxito a diferencia de aquellas que se crean fuera, y que la posibilidad de supervivencia se reduce en $30 \%$. Es decir que la importancia radica en el potencial que aporta la incubadora al ser un centro que orienta y asesora a quienes estén interesados en la creación de sus empresas, tomando en consideración la estructuración de un plan de negocio acorde a sus necesidades y que permita la permanencia en el mercado.

Desde los puntos de vista analizados se requiere que las Instituciones de Educación Superior se preocupen de la creación de incubadoras multidisciplinarias, aprovechando sus dominios y experiencias con la finalidad de que sean el puntal del desarrollo empresarial y productivo y sobre todo que se brinde el apoyo científico, tecnológico a la sociedad para que se formen emprendimientos sostenibles y rentables que aporten en la mejor calidad de vida de las personas y se constituyan en generadores de empleo (TapiaCárdenas, et al., 2020).

Es necesario que las Instituciones de Educación Superior (IES), verifiquen los escenarios en los cuales van a surgir las incubadoras de empresas, es decir si se cumplen con las condiciones establecidas por (Arnaut, 2010), el cual considera que para que una universidad sea emprendedora debe poseer las siguientes características: liderazgo y capacidad emprendedora tanto en los estudiantes como el personal inmerso en el tema, resultados que demuestren impacto tanto en personas como en organizaciones, técnicas de aprendizaje innovador hacia el emprendimiento, educación multidisciplinaria que permita resolver problemas o inconvenientes a nivel mundial.

Hoy en día los emprendedores disponen de apoyo profesional para llevar a cabo sus ideas de negocios, con la implementación de nuevos modelos como las Incubadoras de Empresas (IE) mismas que prestan ayuda a los nuevos empresarios para impulsar sus ideas de negocios y convertirlos en emprendimientos que mediante la elaboración de un 


\section{CIENCIAMATRIA \\ Revista Interdisciplinaria de Humanidades, Educación, Ciencia y Tecnología \\ Año VII. Vol. VII. Nº12. Enero - Junio. 2021 \\ Hecho el depósito de ley: pp201602FA4721 \\ ISSN-L: 2542-3029; ISSN: 2610-802X \\ Universidad Nacional Experimental Francisco de Miranda (UNEFM). Santa Ana de Coro. Venezuela \\ María Fernanda Jimbo-Amaya; Edwin Joselito Vásquez-Erazo; \\ Maira Elizabeth Ramón-Naranjo}

Plan de negocios les permita visualizar la gestión misma del emprendimiento, sus requerimientos y proyecciones empresariales que contribuyan con el éxito del negocio.

De acuerdo con (Perez-Palacios, 2009), la formación y la motivación son estrategias implementadas por las universidades a nivel mundial como un mecanismo que permite aumentar la velocidad y el desarrollo de nuevos emprendimientos, es decir que actualmente se encuentran en marcha 2500 incubadoras tanto en países industrializados como en economías en transición cuya finalidad es potenciar el desarrollo global en diferentes áreas estratégicas.

Por otro lado, (Del-Valle, 2014), establece que el objetivo principal de las incubadoras de empresas en el Ecuador es brindar un ambiente propicio compuesto por: espacio físico además de tecnología que sirva de apoyo en sus labores, así como re direccionamiento con la idea incubada para que se pueda transformar en una empresa rentable que permita lograr objetivos establecidos, innovar y crear fuentes de trabajo.

Según Voisey, et al., (2006) existen cinco elementos a tener en cuenta para que una incubadora sea exitosa esto es: "liderazgo emprendedor, servicios con valor agregado, selección de proyectos, éxito a alcanzar, acceso a los recursos financieros y humanos", por tal motivo se puede afirmar que la selección de proyectos constituye un proceso vital dentro de cualquier modelo de incubación.

Cabe destacar que existen limitaciones en cuanto a espacios, y servicios adicionales que pueden requerir una cantidad superior de recursos, afectando directamente el número de proyectos a ser incubados, por tal motivo se realiza una selección de proyectos con mayor potencial mismos que recibirán una asistencia en la incubadora para iniciar y crecer en el mercado. Los procesos de selección deben contener normas y procedimientos claros, formas estandarizadas manejados por el comité de evaluadores que se encuentra conformado por el director de la incubadora, staff interno, consultores y académicos a incubar (Reyes-Díaz, et al., 2019). Está por demás decir que la presencia de incubadoras a nivel de (IES) es fundamental en la formación académica ya que permite a gran parte 


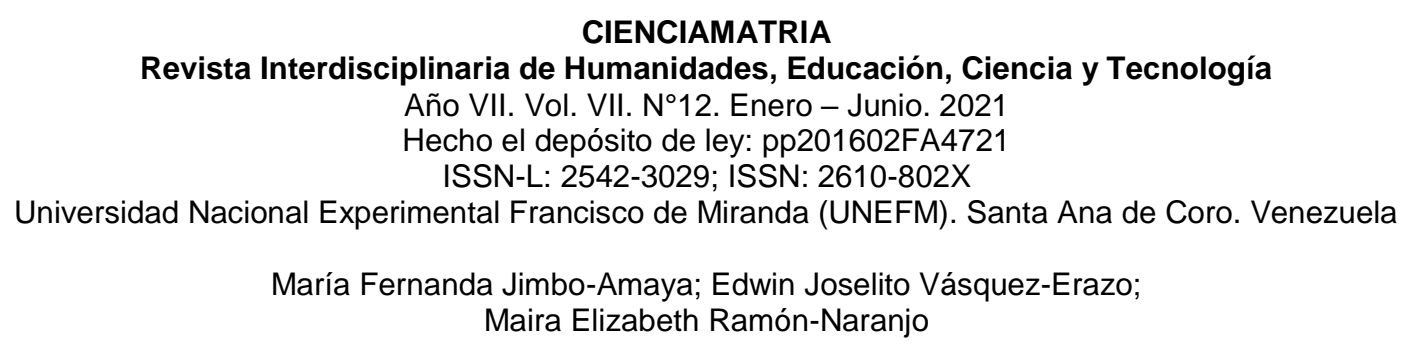

de los estudiantes convertirse en empresarios y generar empleo mejorando las condiciones productivas de la región.

Se puede mencionar también que, la generación de incubadoras empresariales ha permitido el desarrollo y crecimiento en varios países. (Guerra-Triviño, et al., 2015) Indica que con la finalidad de apoyar a pequeños negocios o emprendimientos la Constitución de la Republica en el Ecuador destaca el apoyo hacia las iniciativas de la creación de incubadoras ya que ha permitido mejorar la calidad de vida implementando un sistema económico justo, democrático, productivo sostenible y solidario; (Boza, et al., 2016), manifiesta que se ha detectado baja competitividad e innovación en el tejido empresarial en la última década esto es debido a la frágiles relaciones entre empresas vs universidades. Por consiguiente, las (IES) han cambiado su visión hacia un enfoque de vinculación con la investigación y el sector empresarial.

Según (Risso, et al., 2015), la evolución de las incubadoras ha permitido una estrategia de desarrollo económico, apoyo y promoción a las nuevas empresas que surgen en el mercado hasta conseguir su autonomía. Por otro lado (Ferreiro-Seoane \& CaminoSantos, 2016) considera una ventaja mayor al implementar incubadoras de empresas que consiste en el apoyo integral al emprendedor formando parte de un ecosistema de negocios.

Desde el punto de vista de (Guerra-Triviño, et al., 2015), indican que en el Ecuador el $40 \%$ de emprendimientos viene de la industria minera, agrícola, y el $60 \%$ corresponde a servicios de compra y venta, peluquería, alimentos entre otros, esto quiere decir que se registra un aumento significativo de la tasa de emprendimiento del $26,6 \%$ a diferencia de otros países como Chile que tiene un $23 \%$ y Perú con $20 \%$.

Tal como lo demuestra el informe (GEM , 2019-2020), el cual nos permite observar la facilidad que tienen algunas naciones para crear y gestionar negocios ubicando a Ecuador en el puesto 39 con relación a 54 países participantes. También se puede observar que los países que brinda mayores oportunidades lo ocupan Suiza y Holanda con las posiciones 1 y 2 respectivamente. 


\section{CIENCIAMATRIA \\ Revista Interdisciplinaria de Humanidades, Educación, Ciencia y Tecnología \\ Año VII. Vol. VII. Nº12. Enero - Junio. 2021 \\ Hecho el depósito de ley: pp201602FA4721 \\ ISSN-L: 2542-3029; ISSN: 2610-802X \\ Universidad Nacional Experimental Francisco de Miranda (UNEFM). Santa Ana de Coro. Venezuela \\ María Fernanda Jimbo-Amaya; Edwin Joselito Vásquez-Erazo; \\ Maira Elizabeth Ramón-Naranjo}

\section{Generación de Spin Off Académicas en el seno de las Instituciones de Educación Superior (IES), caracterización y beneficios que aportan con su creación.}

A juicio de (López-Obando, 2017), las nuevas empresas creadas sobre la base del conocimiento y la investigación de docentes universitarios anexos a un centro de investigación, son las denominadas Spin-Off cuyo propósito final es la generación de un nuevo producto/servicio de base tecnológica que contribuya a la sociedad. Las Instituciones de Educación Superior (IES) con la creación de las Spin-Off disponen de uno de los mecanismos de transferencia de conocimientos y tecnología que contribuirán en la creación de nuevas empresas desde la academia. En este sentido las spin-off creadas en las universidades permiten identificar nuevas ideas de negocios promovidas por grupos investigadores desde la universidad, cuya caracterización es la explotación de nuevos procesos, productos/servicios a partir de conocimientos adquiridos desde la academia. Por otro lado (Salazar-Gutierrez, 2017), manifiesta que es un medio que permite transferir ideas creadas y desarrolladas por los estudiantes las cuales nacen mediante conocimientos investigativos adquiridos e impulsados en la academia, con la única finalidad de innovar y crear tecnologías que permitan generar empleos de calidad. En la opinión de (Tamayo, 2011), son empresas que cuentan con el apoyo institucional ya que se generan por profesores, estudiantes o personal que labora en la institución como administrativos, y que su objetivo se traduce en propagar además de aprovechar el conocimiento vinculado con el entorno. Es así que a pesar de todas las oportunidades que brindan las Spin-Off son poco frecuentes en las Instituciones de Educación Superior (IES) por la particularidad hacia la publicación científica dejando de lado la cultura de emprendimiento.

Por otro lado, (Aceytuno \& Paz, 2008), sostiene que para que un proceso emprendedor permita contribuir con la ciencia y tecnología y de paso al surgimiento de nuevos emprendimientos es fundamental contar con el apoyo de las universidades de manera tal que contribuya al cambio de la matriz productiva en el Ecuador. Según (Bravo, et al., 


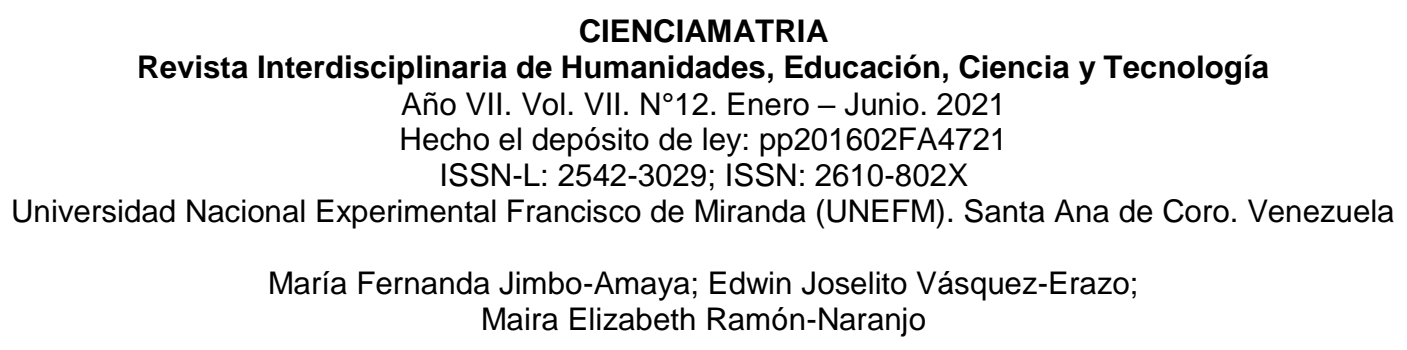

2019), las spin- off poseen características fundamentales para su adecuado desarrollo y están sumadas a los aportes que las universidades realizan en este propósito. Bajo este contexto se fundamenta la labor de las incubadoras de empresas quienes juegan un papel muy importante para la creación de las spin-off y la generación de empleos, al ser organizaciones diseñadas para acelerar el crecimiento y asegurar el éxito de los proyectos emprendedores (Erazo \& Narváez, 2020).

De acuerdo con (Caceres \& Aceytuno, 2015), (Aceytuno \& Paz, 2008), la creación de Spin-Off necesita un compromiso de parte de las instituciones de Educación Superior y que todos sus componentes, misión, visión, políticas y objetivos, así como su cultura organizacional, concomitante a sus funciones sustantivas de docencia, investigación y extensión universitaria que integren dentro sus actividades el desarrollo de las spin-off con la finalidad de propiciar ambientes emprendedores en el interior de las instituciones preservando las normas legales de propiedad intelectual, así como también los principales atributos del emprendedor (Reyes, et al., 2020).

No obstante, es de vital importancia que la universidad sea la encargada de crear y comercializar las ideas que se generen en los centros de innovación, de tal manera que se puedan cristalizar los beneficios obtenidos en pro de la economía de la región (Mosquera, 2015). Es importante destacar que las empresas derivadas Spin-Off, fortalecen su compromiso de colaboración continua con el estado y la organización privada, realizando estudios a partir de la academia que permitan generar nuevos productos/servicios

\section{MÉTODO}

La investigación se realizó en base a una metodología descriptivo con diseño de campo no experimental, como herramienta principal en el levantamiento de la información se ha utilizado las encuestas vía google forms que consta de un cuestionario de 10 preguntas con varias opciones de respuesta en la escala de Likert, la muestra para la investigación realizada estuvo conformada por 17 docentes de la Unidad Académica de Administración, 
con la finalidad de conocer su apreciación en cuanto a la implementación de incubadoras de empresas en las Instituciones de Educación Superior, la información recoilada fue analizada mediante estadística descriptiva (Rodríguez-Jiménez \& Pérez-Jacinto, 2016).

\section{RESULTADOS}

Esta investigación toma como base los resultados de la encuesta, dirigida a la planta docente de Unidad Académica de Administración de la Universidad Católica de Cuenca, misma que para su mayor claridad se ha procedido a dividir en dimensiones, siendo estas:

\section{Dimensión: Emprendimiento desde las universidades}

En lo referente emprendimientos el $75 \%$ de los docentes manifestó que el emprendimiento debe incorporarse en las mallas académicas debido a su importancia, en tanto que el $18 \%$ considera importante desarrollar iniciativas relacionadas con la gestión de emprendimientos, el $7 \%$ restante no aporta mayor criterio al mostrarse neutral.

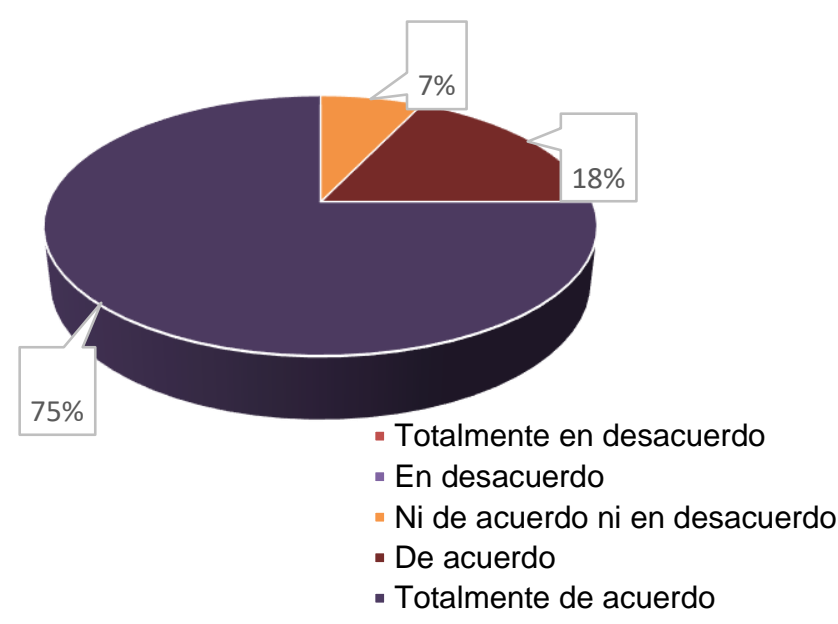

Figura 2. Emprendimiento desde las Universidades. 


\section{Dimensión: Creación de incubadoras de empresas a nivel de Instituciones de Educación Superior (IES).}

El $80 \%$ de los docentes encuestados apuesta por la implementación de incubadoras ya que aportan beneficios para el futuro emprendedor, el $16 \%$ considera conveniente su implementación no sin antes verificar su adaptación en el entorno, en tanto que un $2 \%$ considera casi nula la implementación de estos centros por la falta de apoyo desde la academia.

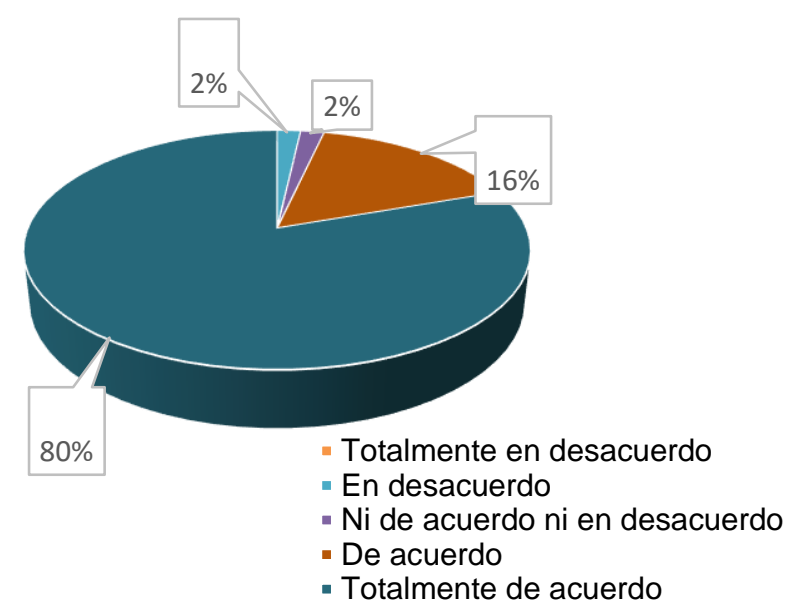

Figura 3. Creación de Incubadoras de Empresas a nivel de (IES).

\section{Dimensión Implementación de Spin-Off en el entorno universitario}

En lo referente a implementación de Spin Off $68 \%$ de los docentes considera que existe un ecosistema adecuado para su generación, en tanto que un $21 \%$ considera que no se cuenta con el apoyo suficiente para su implementación, un $9 \%$ demuestra poco interés en su implementación y un $2 \%$ desconoce los beneficios que aportan con su implementación. 


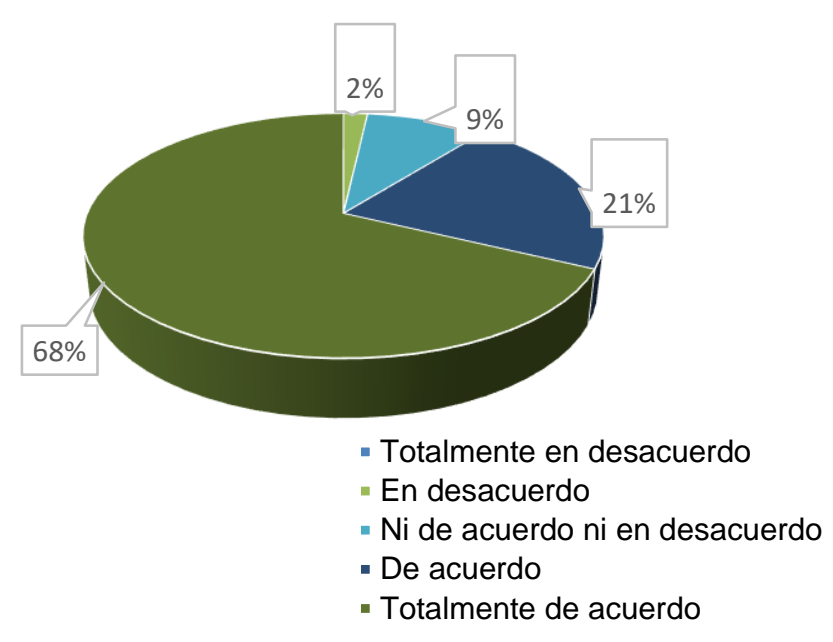

Figura 4. Implementación de Spin-Off en el Entorno Universitario.

Previo a la elaboración de la propuesta resulta de vital importancia realizar un análisis del entorno universitario, mediante una matriz PESTEL misma que permitirá identificar cuáles son los factores que afectan la generación de emprendimiento, es importante mencionar que se ha utilizado la escala de Likert para valorar la importancia del factor $y$ obtener resultados más concretos que nos ayuden a determinar el grado de vinculación que ocupan de acuerdo al factor, los mismos que están divididos de la siguiente manera. 


\section{CIENCIAMATRIA \\ Revista Interdisciplinaria de Humanidades, Educación, Ciencia y Tecnología \\ Año VII. Vol. VII. N¹2. Enero - Junio. 2021 \\ Hecho el depósito de ley: pp201602FA4721 \\ ISSN-L: 2542-3029; ISSN: 2610-802X \\ Universidad Nacional Experimental Francisco de Miranda (UNEFM). Santa Ana de Coro. Venezuela \\ María Fernanda Jimbo-Amaya; Edwin Joselito Vásquez-Erazo; Maira Elizabeth Ramón-Naranjo}

\section{Tabla 1.}

Matriz Pestel.

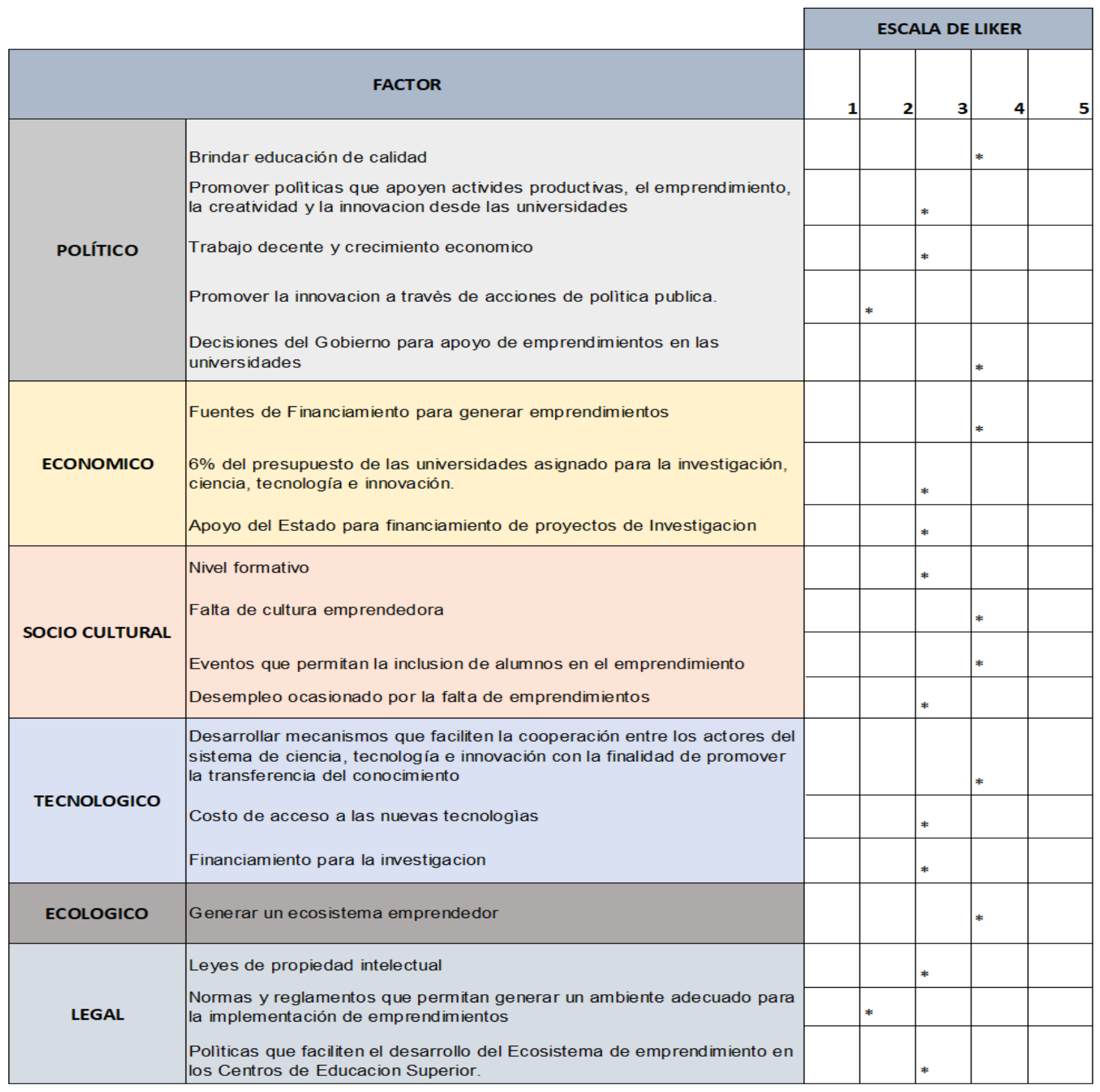




\section{PROPUESTA}

Con la propuesta se pretende analizar y plantear estrategias que contribuyan en la creación de spin off en el entorno universitario, los factores más representativos a tomar en consideración son: docencia, vinculación, investigación, y financiamiento.

\section{SPIN-OFF EN EL ENTORNO UNIVERSITARIO}

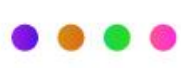

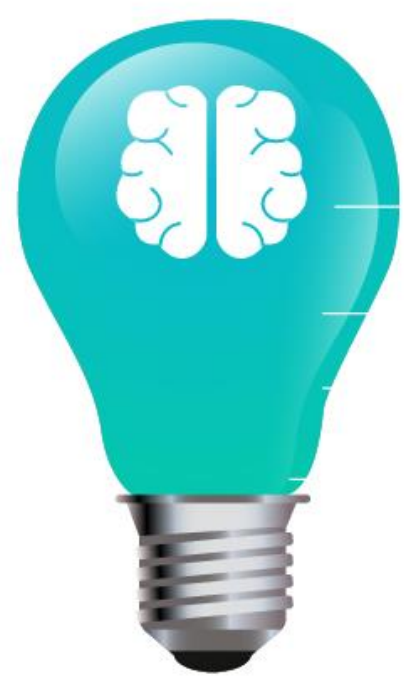
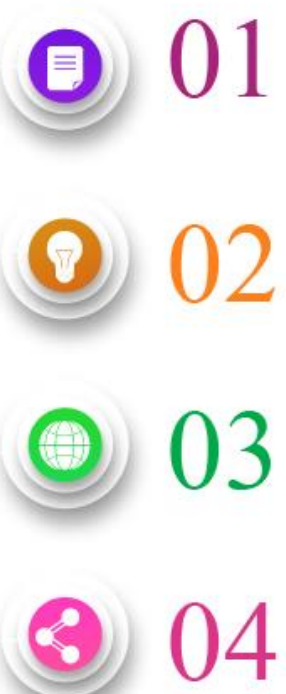

(8) 04
DOCENCIA

VINCULACIÒN

INVESTIGACIÒN

Figura 5. Esquema de la propuesta 


\section{CIENCIAMATRIA \\ Revista Interdisciplinaria de Humanidades, Educación, Ciencia y Tecnología \\ Año VII. Vol. VII. N¹2. Enero - Junio. 2021 \\ Hecho el depósito de ley: pp201602FA4721 \\ ISSN-L: 2542-3029; ISSN: 2610-802X \\ Universidad Nacional Experimental Francisco de Miranda (UNEFM). Santa Ana de Coro. Venezuela \\ María Fernanda Jimbo-Amaya; Edwin Joselito Vásquez-Erazo; \\ Maira Elizabeth Ramón-Naranjo}

\section{Docencia - Vinculación.}

Se ha diseñado una tabla que contiene estrategias, objetivos, resultados y recursos a implementar como se puede ver a continuacion:

\section{Tabla 2.}

Docencia - Vinculación.

\section{Estrategia:}

Implementar cátedras re direccionadas al emprendimiento en el proyecto educativo, integrado al perfil de formación profesional y a los planes y programas de estudio, tomando siempre en consideración la demanda actual de la sociedad y la profesión.

Brindar cierta autonomía a la parte académica para llevar sus ideas a la práctica empresarial dentro del campo de investigación.

\section{Objetivo:}

Se pretende incorporar en los estudiantes métodos y técnicas orientadas al emprendimiento innovador.

Aportar con el conocimiento para buscar estrategias y solucionar

\section{Docencia} problemas empresariales

\section{Resultado:}

Generación de ideas de negocios que contribuyan con la economía de la sociedad.

Mejor sustentabilidad para las empresas

\section{Recursos:}

Implementación de tecnología

Conocimiento e investigación

\section{Estrategia:}


Intercambio de saberes de los emprendedores (know how), y los conocimientos de la academia.

Capacitación continua a docentes y estudiantes en modelos de negocios nuevos e innovadores

Buscar las necesidades sociales en las comunidades en las que se pueda apuntar a generar una microempresa.

Vinculación Generar lazos de cooperación entre la universidad y los sectores productivos.

Propiciar la transferencia tecnológica para crear emprendimiento que se integre al proceso de incubadoras.

\section{Objetivo:}

Ganar, ganar de los docentes y estudiantes por cuanto se aprende de la práctica y los emprendedores fortalecen sus emprendimientos en base de los resultados de la investigación científica.

Satisfacción de las necesidades sobretodo de los sectores que necesiten más apoyo en cuanto a emprendimiento se refiere.

\section{Resultado:}

Mejorar los indicadores

Generar microempresas que ofrezcan productos y servicios de calidad.

\section{Recursos:}

Prácticas de servicio comunitario, laboratorios de tecnología.

Recursos Humanos y financieros. 


\section{CIENCIAMATRIA \\ Revista Interdisciplinaria de Humanidades, Educación, Ciencia y Tecnología \\ Año VII. Vol. VII. N¹2. Enero - Junio. 2021 \\ Hecho el depósito de ley: pp201602FA4721 \\ ISSN-L: 2542-3029; ISSN: 2610-802X \\ Universidad Nacional Experimental Francisco de Miranda (UNEFM). Santa Ana de Coro. Venezuela \\ María Fernanda Jimbo-Amaya; Edwin Joselito Vásquez-Erazo; \\ Maira Elizabeth Ramón-Naranjo}

\section{Investigación - Financiamiento}

En tanto que para la Investigación y financiamiento resulto necesario partir desde una matriz PHVA, misma que permite alcanzar una mejora continua en las áreas descritas partiendo desde la aplicación de cuatros pasos tal como se puede visualizar a continuación.

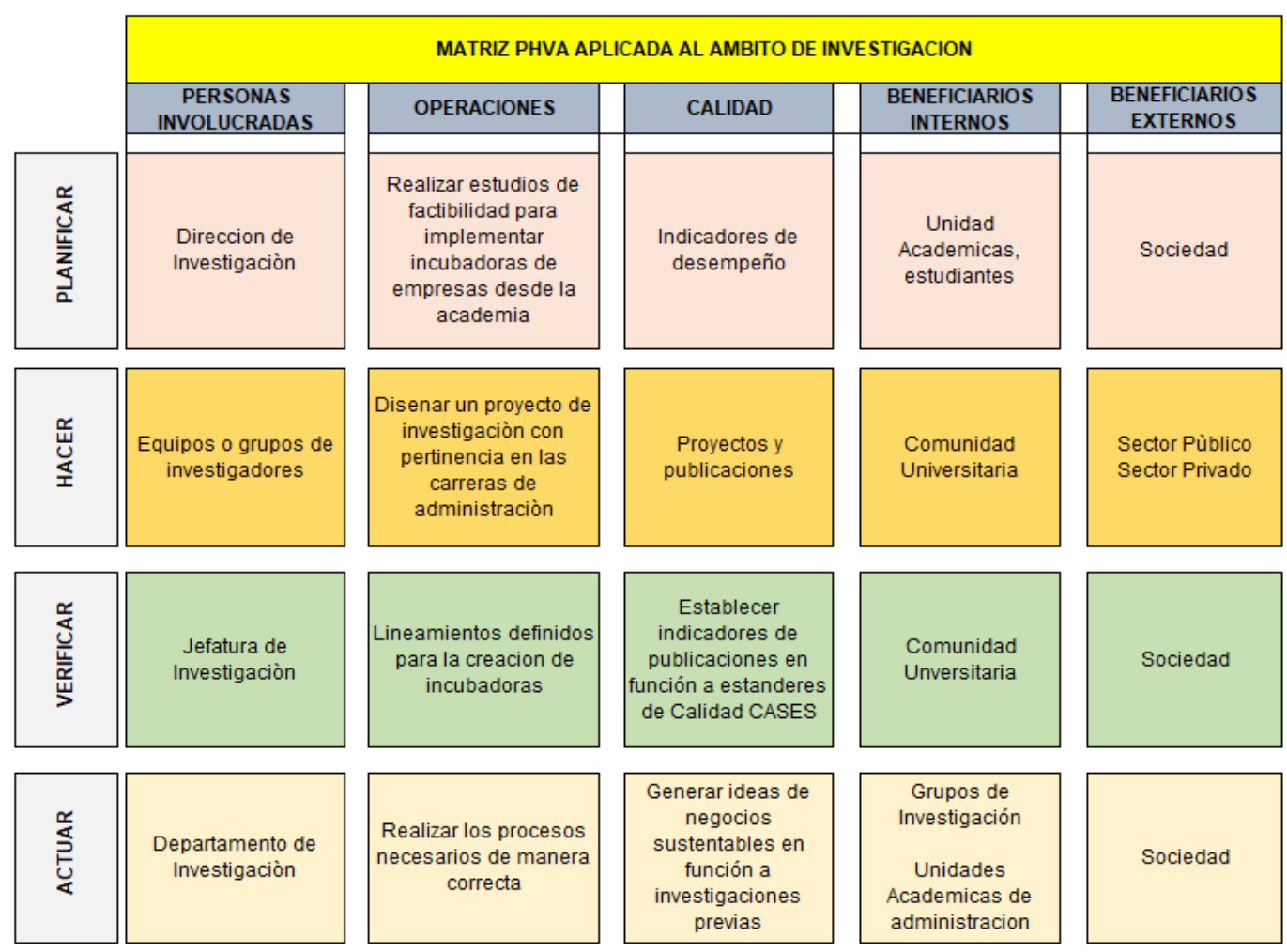

Figura 6. Investigación. 
CIENCIAMATRIA

Revista Interdisciplinaria de Humanidades, Educación, Ciencia y Tecnología

Año VII. Vol. VII. N¹2. Enero - Junio. 2021

Hecho el depósito de ley: pp201602FA4721

ISSN-L: 2542-3029; ISSN: 2610-802X

Universidad Nacional Experimental Francisco de Miranda (UNEFM). Santa Ana de Coro. Venezuela

María Fernanda Jimbo-Amaya; Edwin Joselito Vásquez-Erazo;

Maira Elizabeth Ramón-Naranjo

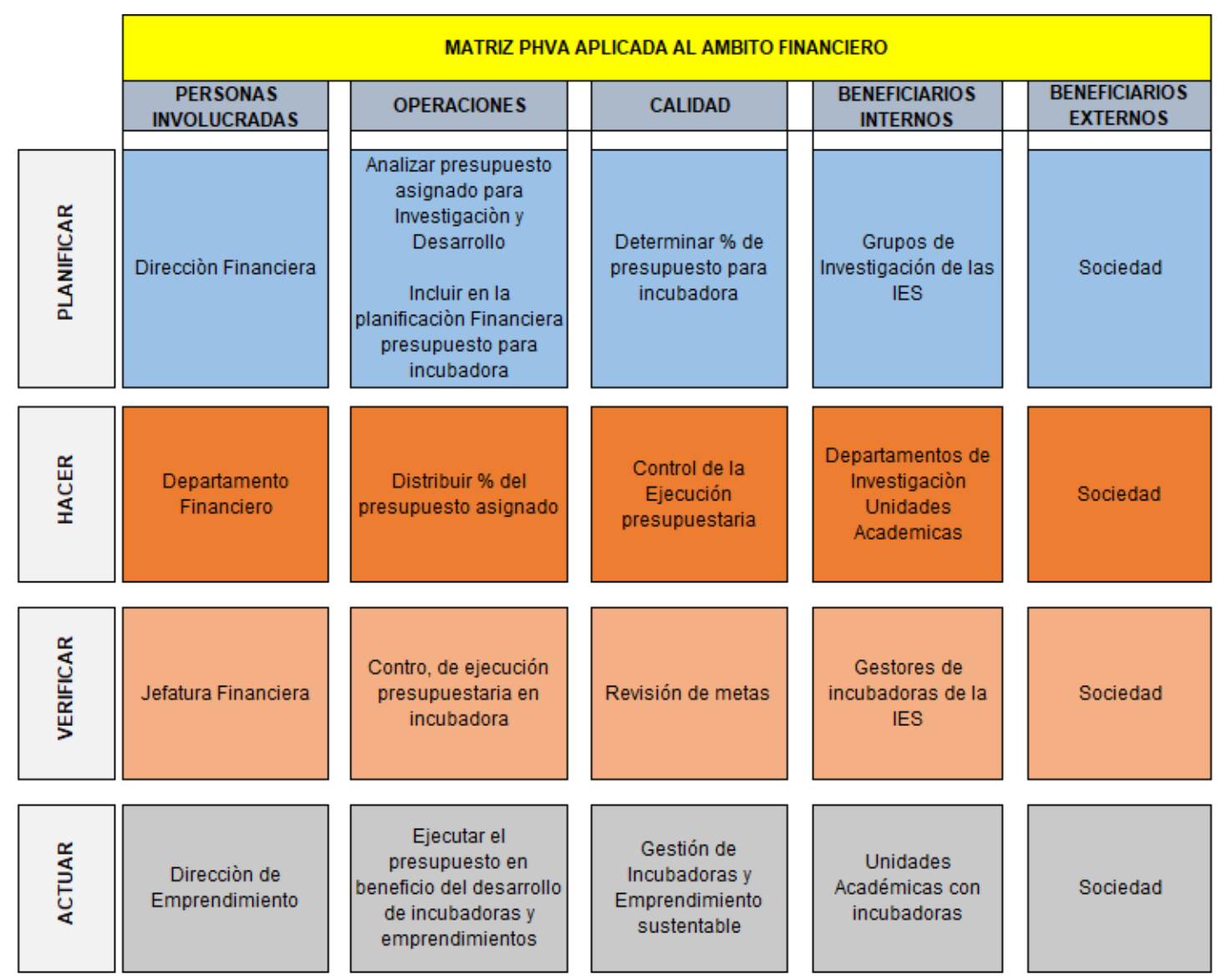

Figura 7. Financiamiento.

\section{CONCLUSIONES}

Una vez concluida la investigación se puede analizar que las Instituciones de Educación Superior (IES) cuentan con una estructura definida para generar emprendimientos pero que depende en gran medida del apoyo con el que estas cuenten en referencia a infraestructura, tecnología, docencia.

Es importante incentivar el emprendimiento desde la academia, esto es mediante la ejecución de proyectos presentados por estudiantes en apoyo conjunto con docentes investigadores los mismos que permitirán desarrollar competencias en su desempeño profesional, es importante recalcar que una universidad necesita desarrollar procesos innovadores en su formación para ser considerada emprendedora. 


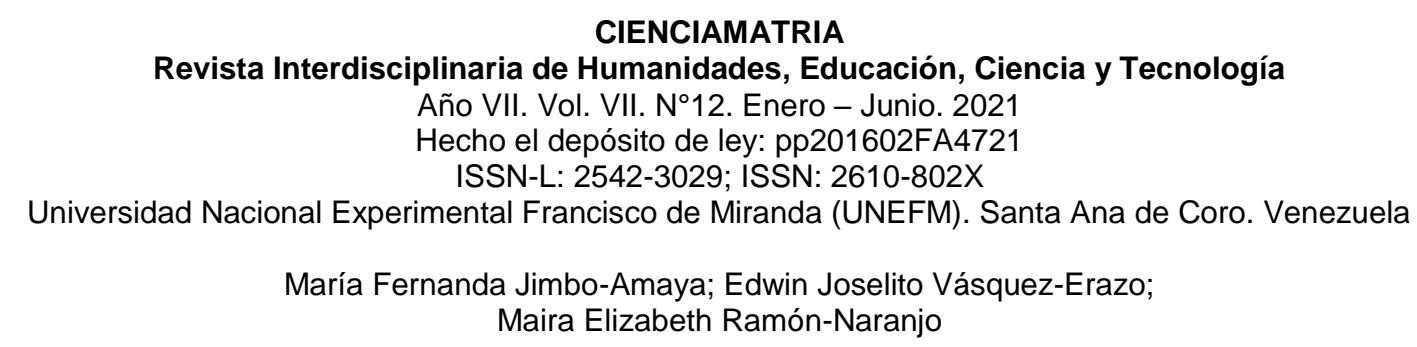

Se hace necesario la creación de incubadoras en las Instituciones de Educación Superior (IES) con la finalidad de gestar las ideas innovadoras de emprendimiento y apoyar en el desarrollo y aceleramiento de los emprendimientos.

\section{REFERENCIAS CONSULTADAS}

Aceytuno, M., \& Paz, M. (2008). La creación de spin-off universitarias: el caso de la Universidad de Huelva [The creation of university spin-offs: the case of the University of Huelva]. Economia Industrial, 91-111. http://hdl.handle.net/10272/10810

Arata-Andreani, A., \& Soto-Salazar, F. (2010). Spin-Off Universidad Emprendedora [SpinOff Entrepreneurial University]. Valparaiso-Chile: Editorial Universidad Técnica Federico santamaría.

Arnaut, D. (2010). Towards an entrepreneurial university. International journal of EuroMediterranean Studies, 3(1), 135-152.

Beltrán-Ibarra, A., \& Lagarda-Leyva, E. (2015). Propuesta de un modelo de vinculacion para una Universidad basada en la Triple Helice [Proposal of a linkage model for a University based on the Triple Helix]. Revista Global de Negocios, 3(6), 45-62.

Boza, J., Tachong, L., \& Moreira, M. (2016). La Universidad como catalizador del desarrollo local en el Ecuador [The University as a catalyst for local development in Ecuador]. Revista Caribeña de Ciencias Sociales, 1-8.

Bravo, S., Benavides, J., Wagner, M., \& Londoño, J. (2019). Perspectivas de las spin-off académicas,como modelo de emprendimiento en las universidades colombianas [Perspectives of academic spin-offs as a model of entrepreneurship in Colombian universities]. Desarrollo Gerencial 11(1), 131-156. https://doi.org/10.17081/dege.11.1.3443

Caceres, R., \& Aceytuno, M. (2015). Academic spin-offs incubation strategies: the case of the Andalusian region. Cuadernos de Gestion, Vol 15, 113-142.

Campos, G., \& Sánchez, G. (2006). La vinculacion universitaria y sus interpretaciones [The university link and its interpretations]. Revista Ingenierias Vol IX N 30, 18-25. 
Del-Valle, M. (2005). Forjando emprendedores. Las incubadoras de empresas en el Ecuador. Serie Capacidad Emprendedora, Caracas: CAF. http://scioteca.caf.com/handle/123456789/453

Díez-Sáez, A. (2014). La Spin-off Universitaria como mecanismo para la transferencia de conocimiento [The University Spin-off as a mechanism for the transfer of knowledge]. Tesis de Grado. Universidad del Pais Vasco, Bilbao. Recuperado de https://n9.cl/y5lh.

Erazo, J. C., \& Narváez, C. I. (2020). La gestión del capital intelectual y su impacto en la efectividad organizacional de la industria de cuero y calzado en la Provincia de Tungurahua - Ecuador [The management of intellectual capital and its impact on the organizational effectiveness of the leather and footwear industry in the Province of Tungurahua - Ecuador]. Revista Espacios, 41(21), 254-271.

Etzkowitz, H., \& Leydesdorff, L. (2000). The dynamics of innovation: from National Systems and "Mode 2" to a Triple Helix of university-industry-government relations. Research Policy, 29(2), 109-123. https://doi.org/10.1016/S0048$\underline{7333(99) 00055-4}$

Ferreiro-Seoane, F., \& Camino-Santos, M. (2016). Analisis Cuatitativo de los Centros de Iniciativas Empresariales medido a travez del metodo de la balanza fiscal: El caso Gallego. [Quantitative Analysis of the Centers for Entrepreneurial Initiatives measured by the fiscal balance method: The Galician ]. Revista Galega de Economia, 25(1), 61-63.

Freire, M. T. (1). Educación en emprendimiento. Revisión de la literatura económica [Education in entrepreneurship. Review of the economic literature]. Iberian Journal of the History of Economic Thought, 2(2), 82-100. https://doi.org/10.5209/rev IJHE.2015.v2.n2.52052

GEM. (2019-2020). Global Entrepreneurship Monitor (GEM) Ecuador 2019-2020. Ecuador: ESPAE.

Guerra-Triviño, O., Hernández-Castillo, D., \& Triviño-Ibarra, C. (2015). Incubadora de Empresas: Via para el emprendimiento en las Universidades [Business Incubator: A path for entrepreneurship in Universities]. Revista Universidad y Sociedad, 7(1), 110-114. 


\begin{abstract}
CIENCIAMATRIA
Revista Interdisciplinaria de Humanidades, Educación, Ciencia y Tecnología

Año VII. Vol. VII. Nº12. Enero - Junio. 2021

Hecho el depósito de ley: pp201602FA4721

ISSN-L: 2542-3029; ISSN: 2610-802X

Universidad Nacional Experimental Francisco de Miranda (UNEFM). Santa Ana de Coro. Venezuela

María Fernanda Jimbo-Amaya; Edwin Joselito Vásquez-Erazo;

Maira Elizabeth Ramón-Naranjo
\end{abstract}

Kaufman, R. (2004). Planificacion Mega, Herramientas Practicas para el Exito Organizacional [Mega Planning, Practical Tools for Organizational Success]. España: Editorial Universitat Jaume, ISBN: 8480214635.

León-Pérez, J. C. \& Benítez-Pincay, L. (2019). Las incubadoras de empresas, una alternativa para el desarrollo de emprendimientos [Business incubators, an alternative for the development of ventures]. Revista de Investigacion Formativa: Innovacion y Aplicaciones Tecnico-Tecnologicas, 1(1), 72-83.

López-Obando, P. (2017). Surgimiento de empresas catalogadas como Spin Off universitarias en Colombia, analisis desde la gerencia de proyectos (fase I) [Emergence of companies classified as university spin-offs in Colombia, analysis from project management (phase I)]. Revista Escuela de Administracion de Negocios, (82), 61-72. https://doi.org/10.21158/01208160.n82.2017.1649

Maridueña-Arroyave, M., Chávez-Ferreiro, J., González-Garcilazo, M., \& NarváezVásquez, G. (2016). Las Spin Off Universitarias: Revision de la literatura sobre la ambiguedad del constructo [ University Spin Off: Literature review on the ambiguity of the construct]. Revista Global de Negocios, 4(7), 95-108.

Martínez-Ortíz, F., Bajaña-Zajia, J., Chávez-Pirca, C., Guerrero-Tipantuña, M., \& OñaSinchiguano, B. (2016). Ecosistema del Emprendimiento en la Universidad Contemporanea [Ecosystem of Entrepreneurship in the Contemporary University] Didasc@lia: Didáctica y Educación, VII(6), 249-262.

Moposita-Yanchatuña, E., Rivera-Badillo, L. \& Morales-Urrutia, D. (2018). El emprendimiento desde la perspectiva de las incubadoras de empresas: un análisis comparado por países [Entrepreneurship from the perspective of business incubators: a comparative analysis by country]. Boletin de Coyuntura, $O(18), 8-13$. http://dx.doi.org/10.31164/bcoyu.18.2018.671

Mosquera, J. (2015). Empresas derivadas "Spin-Off" como elemento emprendedor de las iniciativas empresariales desde las Universidades en la ciudad de Guayaquil. [Spin-off companies as an entrepreneurial element of business initiatives from the universities in the city of Guay. Tesis de Grado (Maestria en Administracion de Empresas). Universidad Catolica Santiago de Guayaquil, Guayaquil-Ecuador. Obtenido de http://repositorio.ucsg.edu.ec/handle/3317/4010 
Perez-Palacios, E. (2009). La Universidad en la formacion de Emprendedores Empresariales y el Apoyo en la creacion de nuevas Empresas [The University in the training of Business Entrepreneurs and Support in the creation of new Companies]. Gestión En El Tercer Milenio, 12(23), 61 - 65.

Reyes, P. E., Narváez, C. I., Erazo, J. C., \& Giler, L. V. (2020). Configuración del impuesto a la patente municipal con base al ingreso de las actividades económicas. Caso: GAD Municipal de Pucará - Ecuador. Revista Espacios, 41(21), 197-211.

Reyes-Díaz, S., Souto-Anido, L., \& Rodríguez-Martínez, J. (2019). El proceso de selección de proyectos en las incubadoras de empresas. Propuesta de procedimiento para una incubadora universitaria cubana. [The project selection process in business incubators Procedure proposal for a Cuban university incubator]. GECONTEC: Revista Internacional De Gestión Del Conocimiento Y La Tecnología, 7(2), 20-42.

Risso, M., Tottereau, L., \& Ramassotti, E. (2015). Proyecto de incubación de pequeñas unidades productivas enmarcadas en los valores de la economía social y solidaria. [Project for the incubation of small productive units framed in the values of the social and solidarity economy]. Ingenieria Solidaria, 11(18), 87-95. http://dx.doi.org/10.16925/in.v11i18.994

Rodríguez-Jiménez, A., \& Pérez-Jacinto, A. (2017). Métodos científicos de indagación y de construcción del conocimiento. Revista Escuela De Administración De Negocios, (82), 175-195. https://doi.org/10.21158/01208160.n82.2017.1647

Romero-Paz, M., Alpízar-Terrero, M., León-Robaina, R., \& Castellanos-Pallerols, G. (2020). Transferencia de resultados de la investigación científica universitaria a través de las incubadoras de empresas [Transference of Scientific Research Results of Universities through Business Incubators]. Retos de la Dirección, 14(1), 235-263.

Salamea, S., \& Peña, S. (2015). Articulaciónde los actores del ecosistema emprendedor como herramienta efectiva para la transición de un proyecto emprendedor a un proyecto comercialmente viable [Articulation of the actors of the entrepreneurial ecosystem as an effective tool for the transition from an entrepreneurial project to a commercially viable project]. Revista de la Facultad de Ciencias Quimicas, 11,16. 


\section{CIENCIAMATRIA \\ Revista Interdisciplinaria de Humanidades, Educación, Ciencia y Tecnología \\ Año VII. Vol. VII. №12. Enero - Junio. 2021 \\ Hecho el depósito de ley: pp201602FA4721 \\ ISSN-L: 2542-3029; ISSN: 2610-802X \\ Universidad Nacional Experimental Francisco de Miranda (UNEFM). Santa Ana de Coro. Venezuela \\ María Fernanda Jimbo-Amaya; Edwin Joselito Vásquez-Erazo; \\ Maira Elizabeth Ramón-Naranjo}

Salazar-Gutierrez, M. (2017). Modelo de Spin-Off de Base Investigativa como impulsor de empresas sostenibles desde la FCECEP [Research-Based Spin-Off Model as a promoter of sustainable enterprises from the FCECEP]. Trabajo de Investigacion (Maestria en Gestion Empresarial). Universidad Libre-Seccional Cali, Santiago de Cali. http://hdl.handle.net/10901/10300

Tamayo, L. (2011). Condiciones Institucionales para la Creacion de una Spin Off Academica: Caso, la Faculta de Minas de la Universidad de Colombia. [Institutional Conditions for the Creation of an Academic Spin Off: The case of the Faculty of Mines of the University of Col]. Tesis de Maestria. Universidad Nacional de Colombia, Medellin. https://n9.cl/37049

Tapia-Cárdenas, H. A., Erazo-Álvarez, J. C., Narváez-Zurita, C. I., \& Matovelle-Romo, M. M. (2020). Estrategias para fomentar el emprendimiento y desarrollo empresarial [Strategies to promote entrepreneurship and business development]. Revista Arbitrada Interdisciplinaria Koinonía, 5(10), 833-861. http://dx.doi.org/10.35381/r.k.v5i10.837

Torres-Vázquez, K. F., Erazo-Álvarez, J. C., Narváez-Zurita, C. I., \& Moreno, V. P. (2020). El emprendimiento juvenil en función al perfil psicosocial y las condiciones del entorno familiar [Youth entrepreneurship according to the psychosocial profile and the conditions of the family environment]. Revista Arbitrada Interdisciplinaria Koinonía, 5(10), 729-756. http://dx.doi.org/10.35381/r.k.v5i10.714

Trippl, M., Sinozic, T., \& Lawton, H. (2015). The Role of Universities in Regional Development: Conceptual Models and Policy Institutions in the UK, Sweden and Austria. European Planning Studies, 23:9, 1722-1740. https://doi.org/10.1080/09654313.2015.1052782

Voisey, P., Gornall, L., Jones, P., \& Thomas, B. (2006). The measurement of success in a business incubation project. Journal of Small Business and Enterprise Development, 13(3), 454-468. https://doi.org/10.1108/14626000610680307 